\title{
Integrated chemiresistor array for small sensor platforms
}

\author{
R. C. Hughes, S. A. Casalnuovo, K. O. Wessendorf, D. J. Savignon, S. Hietala, S. V. Patel and \\ Sandia National Laboratories, Albuquerque, New Mexico, 87185
}

\begin{abstract}
Chemiresistors are fabricated from materials that change their electrical resistance when exposed to certain chemical species. Composites of soluble polymers with metallic particles have shown remarkable sensitivity to many volatile organic chemicals, depending on the ability of the analyte molecules to swell the polymer matrix. These sensors can be made extremely small ( $<100$ square microns), operate at ambient temperatures, and require almost no power to read-out. However, the chemiresistor itself is only a part of a more complex sensor system that delivers chemical information to a user who can act on the information. We present the design, fabrication and performance of a chemiresistor array chip with four different chemiresistor materials, heaters and a temperature sensor. We also show the design and fabrication of an integrated chemiresistor array, where the electronics to read-out the chemiresistors is on the same chip with the electrodes for the chemiresistors. The circuit was designed to perform several functions to make the sensor data more useful. This low-power, integrated chemiresistor array is small enough to be deployed on a Sandia-developed microrobot platform.
\end{abstract}

\section{Introduction}

Sensors for organic solvent vapors are required for the detection of leaks, toxic chemicals, explosives, and solvent spills. As part of a system, these sensors need to be highly sensitive to small concentrations of vapors in ambient air, while consuming minimal power for use in portable devices. Such a sensor system must be able to quickly and reproducibly distinguish solvents from the ambient relative humidity, and classify the responses as a particular solvent, relative humidity, a mixture, or unknown. The development of a single sensor to distinguish different solvents is difficult; however, sensor arrays with several sensitive devices can be used to sense a wide variety of solvents. Sophisticated pattern-recognition algorithms can aid in the analysis of signals from several sensors in an array and can be used to determine the class of analyte measured.[1-3] A significant amount of research has been performed to develop sensor arrays comprised of several sensitive elements.[4]

In this paper we discuss chemiresistor arrays fabricated from inks that are solutions of high molecular weight polymers mixed with carbon particles. The preparation of the Sandia inks and the performance of many individual chemiresistors have been presented in several publications [5, 6. 7]. Other groups have reported data on similar chemiresistors.[2] If a polymer/conductive particle composite increases its volume by thermal expansion or by swelling when absorbing a chemical, the electrical resistance increases due to the breaking of some of the conductive pathways through the film. The expansion can produce large increases in resistance if the polymer volume is changed close to the percolation threshold [8-10]. This threshold concentration has been found to be between 20 and $40 \%$ by volume of the conductive particles. The response of these composite films to different solvents depends on the particular solvent-polymer interaction, while the conductive particles only report the degree of swelling. [5-7] Such materials have been modeled as a network of resistors and diodes, where resistors represent the conductive network of carbon particles and diodes represent the polymer-filled dielectric gaps between the particles.[11]

The degree of swelling of a particular polymer is related to its solubility parameter and the solubility parameter of the solvent. The solubility parameter can be used to describe the free energy of mixing for solvents and polymers. Two solvents that have identical solubility parameter values will form ideal solutions and will have almost zero heat (enthalpy) of mixing. Such ideal solutions of two liquids follow Raoult's law: the vapor pressure of each of the solvents is proportional to the mole fraction of the solvent in the liquid phase. The same rules apply for solvent-polymer mixtures where the amount of solventinduced polymer swelling depends on the partitioning of the solvent vapor into the polymer film. The lowest detectible concentration of a particular Volatile Organic Chemical (VOC) depends largly on its vapor pressure at ambient temperatures. Very low vapor pressure VOC's can usually be detected at the ppm level if the best polymer for the chemiresistor is chosen. [5-7] 


\section{DISCLAIMER}

This report was prepared as an account of work sponsored by an agency of the United States Government. Neither the United States Government nor any agency thereof, nor any of their employees, make any warranty, express or implied, or assumes any legal liability or responsibility for the accuracy, completeness, or usefulness of any information, apparatus, product, or process disclosed, or represents that its use would not infringe privately owned rights. Reference herein to any specific commercial product, process, or service by trade name, trademark, manufacturer, or otherwise does not necessarily constitute or imply its endorsement, recommendation, or favoring by the United States Government or any agency thereof. The views and opinions of authors expressed herein do not necessarily state or reflect those of the United States Government or any agency thereof. 


\section{DISCLAIMER}

Portions of this document may be illegible in electronic image products. Images are produced from the best available original document. 
Use of the solubility parameter and the idea of partitioning of the solvent between two phases have already been studied for determining the relative responses of gas sensor arrays.[2, 5-7,12] Since it is unlikely that a specific polymer will be sensitive to only one solvent (every polymer absorbs a number of solvents having similar solubility parameters), an array of sensors is an effective means to discriminate against interfering vapors. A common and obvious source of interference is relative humidity in the ambient environment. Water vapor has been found to change the relative sensitivity of certain polymers to solvent vapors and the patterns of responses obtained from arrays containing those polymers.[7,13] To build a sensor array that is capable of identifying the maximum number of analytes, the array should contain several different sensors that are as chemically varied as possible, with at least one sensor having significant sensitivity to relative humidity.

\section{Experimental Details}

\section{Chemiresistor Array}

A compact array of four different chemiresistors was fabricated on a silicon chip along with two heaters and temperature sensor. A photograph of one of the arrays is shown in Fig. 1. The electrode patterns, temperature sensor, and heaters were made of platinum and titanium created using standard photolithographic techniques on a silicon wafer with a thick insulating oxide layer. The platinum $(1000 \AA)$ on titanium $(200 \AA)$ features were deposited using evaporation. The low resistance heater strips are at either end of the sensor chip and the temperature sensor is a higher resistance meander line in the center (about $900 \mathrm{ohms}$ ). The temperature sensor has a TCR (temperature coefficient of resistance) value of $0.002^{\circ} \mathrm{C}^{-1}$. The electrode patterns for the chemiresistors provide for a four-terminal measurement of the resistance with two different spacings for the inner (voltage sensing) electrodes: 50 microns and 100 microns. There is a pair of the two electrode spacings on each side. When the mask was designed it was not known if the spacing would have any significant impact on the chemiresistor performance. It turns out that no spacing effects have been discovered for these wide spacings, but earlier electrode arrays with spacings of less than 10 microns had problems with shorting by agglomerated carbon "clumps". Over 130 sensor arrays can be fabricated on a typical 3 inch diameter wafer. Each different sensor type can be formed on many arrays sequentially, in a batch process mode.

The four different sensors in this particular array were formed from inks as described in Refs. [5-7] for the following polymers: PNVP poly(n-vinyl pyrrolidone), PVA poly(vinyl alcohol), PEVA poly(ethylene-vinyl acetate) and PIB poly(isobutylene). The sensor electrodes were coated with the chemiresistive inks using an Asymtek Century Series C-702 Automated Dispensing Unit. The 740 V Low Viscosity attachment was used with a 27 Gauge, 1/2" needle. The inks were applied to the sensor at a rate of 1.5 inches per second, resulting in a line approximately 500 microns wide by 2300 microns long. The samples were dried at room temperature. The final dimensions (thickness, width, and length) of the deposited film are highly dependent on the deposition rate and the viscosity of each ink.

This chip has been mounted in a 16-pin DIP package with the dimensions of about $2 \mathrm{~cm}$ by $0.7 \mathrm{~cm}$ as seen in Fig. 1 . It can then be inserted in a remote header with ribbon cable to a printed circuit board where the individual resistances can be measured along with the chip temperature. The chip temperature can be controlled above ambient by using a feedback circuit to apply current to the heaters, or for lower power operation at ambient temperature, the chemiresistors can be temperature compensated from their measured TCR values.

\section{Response of the Chemiresistor Array to Volatile Organic Chemicals}

The array was wire bonded into a 16-pin DIP package, which could be inserted in a specially designed test fixture. The fixture was attached to a vapor delivery system that had the capability of mixing several gases as well as two solvent vapor streams, using nitrogen as the carrier gas through gas washing bottles ("bubblers"). The bubblers have a wide, porous ceramic frit at the base. Carrier gas is introduced into the solvent through a side arm and up through the frit. The solventsaturated gas mixture exits the bubbler at the top and is then mixed with pure nitrogen to adjust the solvent concentration as desired. Mass flowmeters were used to control the composition of the gas streams, and a constant total flow rate of 1000

$\mathrm{cm}^{3} / \mathrm{min}$ was used for all experiments. The test fixture was placed in a constant-temperature chamber, that was maintained at $21.4^{\circ} \pm 0.3^{\circ} \mathrm{C}$. A digital multimeter (7.5 digit resolution) was used to measure the composite-film resistances. The test gases were passed through a coil of tubing inside the constant-temperature chamber and upstream of the test fixture. This ensured that the inlet gas stream was thermally equilibrated with the sensors. Data acquisition was performed using LabVIEWTM software on a personal computer to control the flow controllers and acquire data from the multimeter. 
Figure 2 shows the response of three of the four chemiresistors on one of these arrays to a series of pulses of VOC vapors. Dry $\mathrm{N}_{2}$ flows before and after each pulse of VOC. In each case, the concentration, or partial pressure, of the vapor is $10 \%$ of the saturated vapor pressure at the bubbler temperature, about $21^{\circ} \mathrm{C}$. The reason for using this concentration is given in detail in our previous papers, Refs [5-7], but it involves normalizing out the effect of entropy on the solubility of the vapors in these polymers. If there were no chemical interactions of the vapors with the polymers (i.e. zero heat of mixing), all the signals would be the same size from entropy considerations. The relative differences in the responses then gives a better idea of the relative heats of mixing. By comparing the signals from the three sensors for the various VOCs, it is easy to see that the PVA sensor is more sensitive to polar molecules like water and methanol, while the PEVA sensor more sensitive to less polar molecules.

Figure 3 shows a "Globe" plot of the data in Fig. 2 to demonstrate how one VOC can be distinguished from another by the relative responses. These plots are discussed in our previous papers, but basically they depict a3-dimensional view of a vector formed from the three signals from the sensors. The vectors are equalized so that each vector has the same length, i.e. each one extends out to the surface of the unit sphere. For example, the strong response of PEVA to trichloroethylene (TCE), places the response vector close to the lower left corner. As pointed out in Refs. [3,5-7], the "VERI"[3] pattern recognition algorithm performs a similar operation to the human vision used in viewing Fig. 3 and identifies the VOCs by the clustering of the unknown data points with training data from the known VOCs. Examples of this identification technique, using individual sensors for both pure and binary compounds of many different concentrations is given in Refs. [3, 5-7].

\section{Analog Chemiresistor ASIC}

The chemiresistor array shown in Fig. 1 is quite small and combines the sensors with a chip heater and temperature sensor. But it still requires read-out electronics and controls. A printed circuit board with surface mounted components could provide those functions, but it would require at least a $10-\mathrm{cm}$ by $10-\mathrm{cm}$ board. This size is too large for most micro-robot applications, and this specification led to the design and fabrication of an aluminum-based ASIC (Application Specific Integrated Circuit) to perform these functions on a very small footprint. The ASIC was fabricated at AMI using their BiCMOS process. This process allows for the use of NPN bipolar along with standard CMOS transistors to make an analog design with a high degree of design flexibility and performance. The integrated chemiresistor ASIC also allows multiple sensors to be fabricated on a single substrate. A photo of the four sensor ASIC array is given in Fig. 4. In this design each chemiresistor is connected to its own analog electronics. This was done so each chemresistor could be individually temperature compensated by the on-chip electronics, which use individual calibrations for dc set point and temperature compensation. Having the chemiresistor and electronics co-located with the integrated circuit minimizes undesired temperature responses by using the reduced size to minimize any cross chip temperature gradients. The bonding (I/O) pads are arranged around the edges to make it easier to wirebond the whole array to a substrate. Future improvements in the ASIC circuit will include the use of digital pots to perform the chemiresistor baseline corrections and temperature compensation. The current ASIC design and footprint allow room for additional circuitry if needed.

A photomicrograph of one chemiresistor ASIC diced out of the wafer is shown in Fig. 5. The chemiresistor itself is on the left side covering most of the four parallel electrodes. The outer, current injecting electrodes were made wider to facilitate charge injection, while the inner, voltage pick-off electrodes were made thinner to give an accurate voltage drop measurement. The bonding pads around the edges allow various test voltages to be measured and provide links to the offchip voltages, calibration resistors and the sensor output voltages. The entire ASIC is coated with a except for the bonding pads and regions where the chemiresistor film is deposited. $\mathrm{nm}$ thick layer of silicon nitride,

In addition to the circuit design, which needs to be flexible enough to accommodate a variety of chemiresistor base resistances, TCRs and signal magnitudes, the electrodes for the sensor can not be made from aluminum because of severe contact resistance problems. Wafer level post-processing of the ASIC chemiresistive sensor to deposit a platinum (1000 $\AA)$ on titanium $(200 \AA)$ overlayer, by evaporation, on top of the aluminum electrodes was accomplished using a metal lift-off technique. Clarient AZ 9245 resist was used as a masking material, and the sensor area was exposed using a Karl Suss MA6/BA6 contact aligner. Titanium $(200 \AA)$ and platinum $(1000 \AA)$ were deposited using a Temescal CV-8 metal evaporator.

To help reduce contact resistance effects, a four-point probe measurement is used. The circuit for the four-point measurement is depicted in the following diagram: 


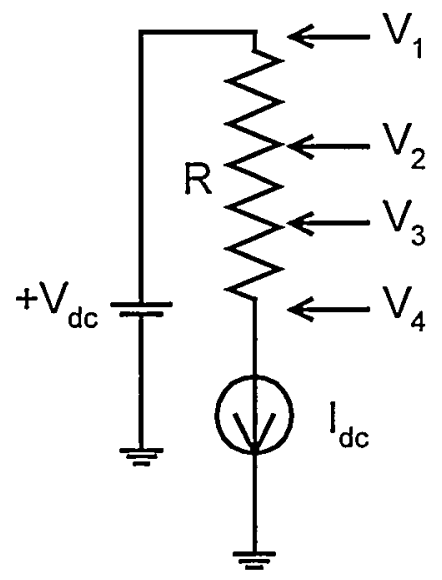

To measure a segment of the resistor, $R$, independent of $V_{d c}$, two voltage measurements $V_{2}$ and $V_{3}$ are made and the incremental resistance, $R_{i}$ is calculated by $R_{i}=\left(V_{2}-V_{3}\right) / I_{d c}$ where $I_{d c}$ is the known current from a current source. For the chemiresistor ASIC, it was desired to have an analog voltage that effectively measures the chemiresistor and provides a convenient output signal for use with commercial or custom telemetry units.

The measurement described here has the following practical limitations: $V_{d c}$ must be greater than $I_{d c}(R)$ due to the fact that the practical current source must be biased at least slightly above ground, and to select a current source value the resistance value must be known before hand.

The method chosen for the ASIC four-point measurement employs a precision voltage controlled current source with a feedback loop that servoes the voltage $V_{2}-V_{3}\left(V_{\text {drop }}\right)$ to a predetermined value, say 0.5 volts. The voltage applied to the voltage controlled-current-source will provide a large dynamic range signal that indirectly measures an incremental part of the chemireistor. This measurement is independent of $V_{d c}$ and allows a temperature compensation scheme in that the value of $V_{\text {drop }}$ can easily be made a linear function of temperature, thus negating the chemiresitor temperature dependence.

The chemiresistor ASIC includes a linear temperature compensation scheme, as seen in Fig. 6 . This circuit uses a voltagecontrolled current source to servo a voltage, $V_{\text {drop }}$, to a desired value, approximately 0.5 volts. The actual value of $V_{\text {drop }}$ is determined by the desired static value of $\mathrm{V}_{\text {out } 1}$ and the value of the calibration resistor and the temperature compensation circuit:

$$
\mathrm{V}_{\text {outl }}=4\left(\mathrm{R}_{\text {cal }}\right)\left(\mathrm{R}_{\text {chem }}\right) /\left(\mathrm{V}_{\text {drop }}-\mathrm{V}_{\text {temp }}\right)
$$

This dc voltage is nominally adjusted to 3.0 volts, which is the nominal value of $\mathrm{V}_{\text {offset }}$.

$$
\mathrm{V}_{\text {out2 }}=10\left(\mathrm{~V}_{\text {out } 1}-\mathrm{V}_{\text {offset }}\right)+\mathrm{V}_{\text {offset }}
$$

Where $R_{\text {cal }}$ is the calibration resistor and $R_{\text {chem }}$ is the incremental value of the chemiresistor. $V_{\text {temp }}$ is a temperature dependent variable used to cancel the temperature dependence of $R_{\text {chem. }}$. This variable can be adjusted for both positive and negative temperature coefficients via select resistors.

The temperature coefficient of the chemiresistor can be negated by the analog circuit through a range of 0 to $\pm 4 \% /{ }^{\circ} \mathrm{C}$. The reference used for this temperature compensation circuit comes from the $-2 \mathrm{mV} /{ }^{\circ} \mathrm{C}$ temperature response of the bipolar transistor $V_{b e}$. This compensation is open-loop and linear to temperature therefore the temperature coefficient of the chemiresistor and its linearity must be known or the substrate operating temperature range must be limited. The output, $V_{\text {out2 }}$, is an amplified $(10 \mathrm{x}) \mathrm{V}_{\text {outl }}$. The circuit baseline is adjusted for $\mathrm{V}_{\text {out1 }}$ and $\mathrm{V}_{\text {our }}$ to be approximately 3.0 volts; accomplished by adjusting the calibration resistor and $\mathrm{V}_{\text {offset, }}$ respectively. These adjustments are performed external to the ASIC using external resistors. The output $\mathrm{V}_{\text {outl }}$ also has the feature of accommodating large dynamic swings in the chemiresistor resistance. As was shown in Refs. [5-7], the chemiresisor resistance change becomes superlinear (almost exponential) at higher concentrations of the VOCs. In fact the resistance will increase several decades as the concentration approaches the saturated vapor pressure of some VOCs. The circuit output compresses these large changes with a loss of resolution, but not 
the saturation effect that occurs with a linear output circuit. The change in resistance over at least two decades can be monitored with this circuit with reasonable resolution.

\section{Integrated Chemical Microsensor Robot}

As an example of the utility of integrated microsensors we show a recently developed Sandia microrobot prototype which uses the integrated chemiresistor. The details of the fabrication of this microrobot and its performance will be given in a future publication. Some of its features are given in Fig. 7. The wheels have been removed from the small drive motors for clarity. The other two corners have casters on them. The substrate for the electronics board is a lithium battery. The IR communications port provides line-of-sight data transfer and commands along with an IR proximity detector to prevent collisions with other objects. One can imagine other types of mobile micro-platforms that could use the integrated chemiresistor ASIC: flying machines, submarines and inconspicuous sensor emplacements.

\section{CONCLUSIONS}

In this paper we have shown how a simple chemical sensor technology, the chemiresistor, can be implemented in a system with very small size and power consumption. The response of the chemiresistor itself has been measured for a wide variety of VOCs, but in a small system the measurement circuitry must be integrated and perform a number of housekeeping functions to provide useful information to the overall system for decision making. We have presented a integrated prototype sensor and have shown how it can fit into a microrobot platform.

\section{ACKNOWLEDGMENTS}

Sandia is a multiprogram laboratory operated by Sandia Corporation, a Lockheed Martin Company, for the United States Department of Energy under Contract DE-AC04-94AL85000. We would like to acknowledge the technical assistance of Mark Jenkins, Jon Blaich and Graham Yelton in the fabrication and testing of the chemiresistor arrays.

\section{REFERENCES}

[1] Freund, M. S.; Lewis, N. S. Proc. Natl. Acad. Sci. USA 1995, 92, 2652.

[2] Lonergan, M. C.; Severin, E. J.; Doleman, B. J.; Beaber, S. A.; Grubbs, R. H.; Lewis, N. S. Chem. Mater. 1996, 8, 2298.

[3] Osbourn, G. C.; Bartholemew, J. W.; Ricco A. J.; Frye, G. C. Acc. Chem. Res. 1998, 31, 297.

[4] Nagle, H. T.; Schiffman, S. S.; Gutierrez-Osuna, R. IEEE Spectrum, 1998, 35, 22.

[5] Eastman, M. P.; Hughes, R. C.; Yelton, W. G.; Ricco, A. J.; Patel, S. V.; Jenkins, M. W. "Application of the Solubility Parameter Concept to the Design of Chemiresistor Arrays," J. Electrochem. Soc. 146, 3907-3913 (1999).

[6] Hughes, R. C.; Eastman, M. P.; Yelton, W. G.; Ricco, A. J.; Patel, S. V.; Jenkins, M. W. Tech. Digest of the 1998 Solid-State Sensor and Actuator Workshop, Transducers Research Foundation: Cleveland, 1998, pp. 379-382.

[7] Sanjay V. Patel, Mark W. Jenkins, Robert C. Hughes, W. Graham Yelton, and Antonio J. Ricco,. "Differentiation of Chemical Components in a Binary Solvent Vapor Mixture Using Carbon/Polymer Composite-Based

Chemiresistors" Anal. Chem In press March 2000. (see ASAP March 3,2000)

[8] Heaney, M. B. Appl. Phys. Lett. 1996, 69, 2602.

[9] M. B. Heaney, Physica A, 1997, 241, 296.

[10] Viswanathan, R.; Heaney, M. B. Phys. Rev. Lett. 1995, 75, 4433.

[11] Hampl, J.; Bouda, V. Synthetic Metals, 1994, 67, 129.

[12] Grate, J. W.; Kaganove, S. N.; Bhethanabotla, V. R. Anal. Chem. 1998, 70, 199.

[13] Zellers, E. T.; Han, M. Anal. Chem. 1996, 68, 2409. 


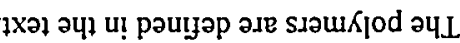

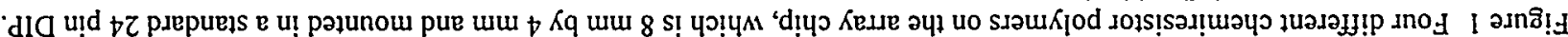

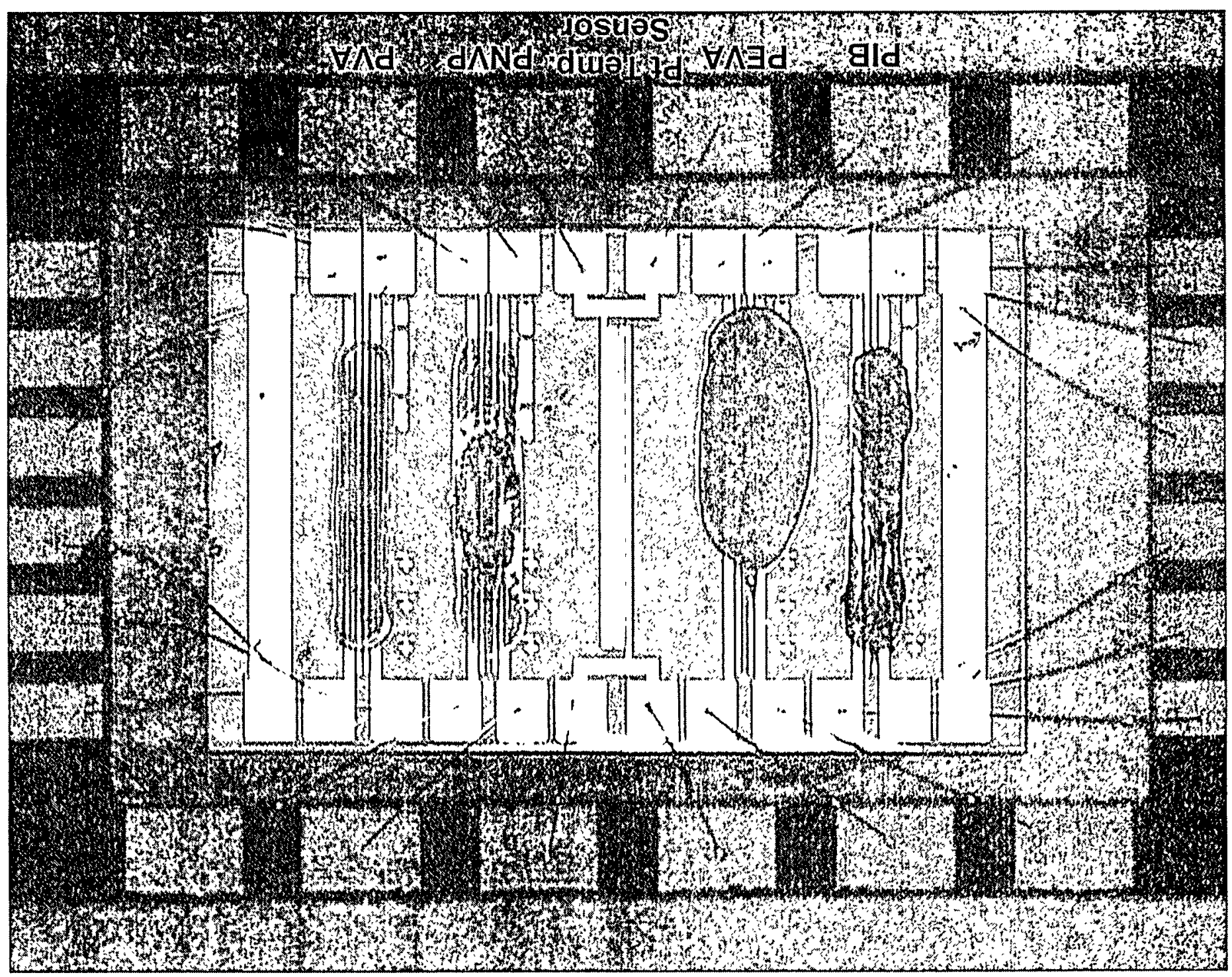



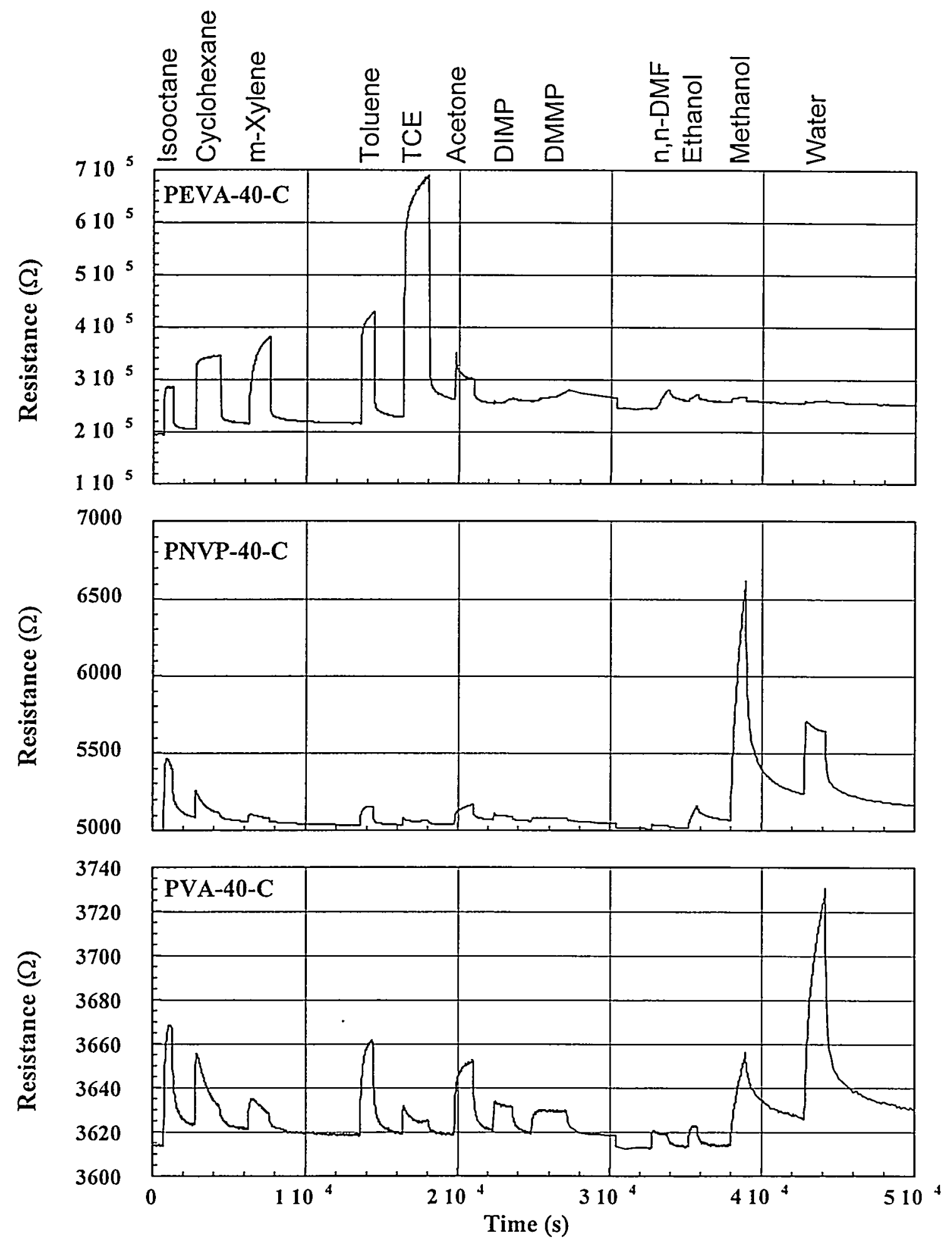

Figure 2 The responses of three of the chemiresistors on the array to a variety of volatile organic chemicals (VOCs). Each pulse of VOC is followed by a dry $\mathrm{N}_{2}$ purge. 


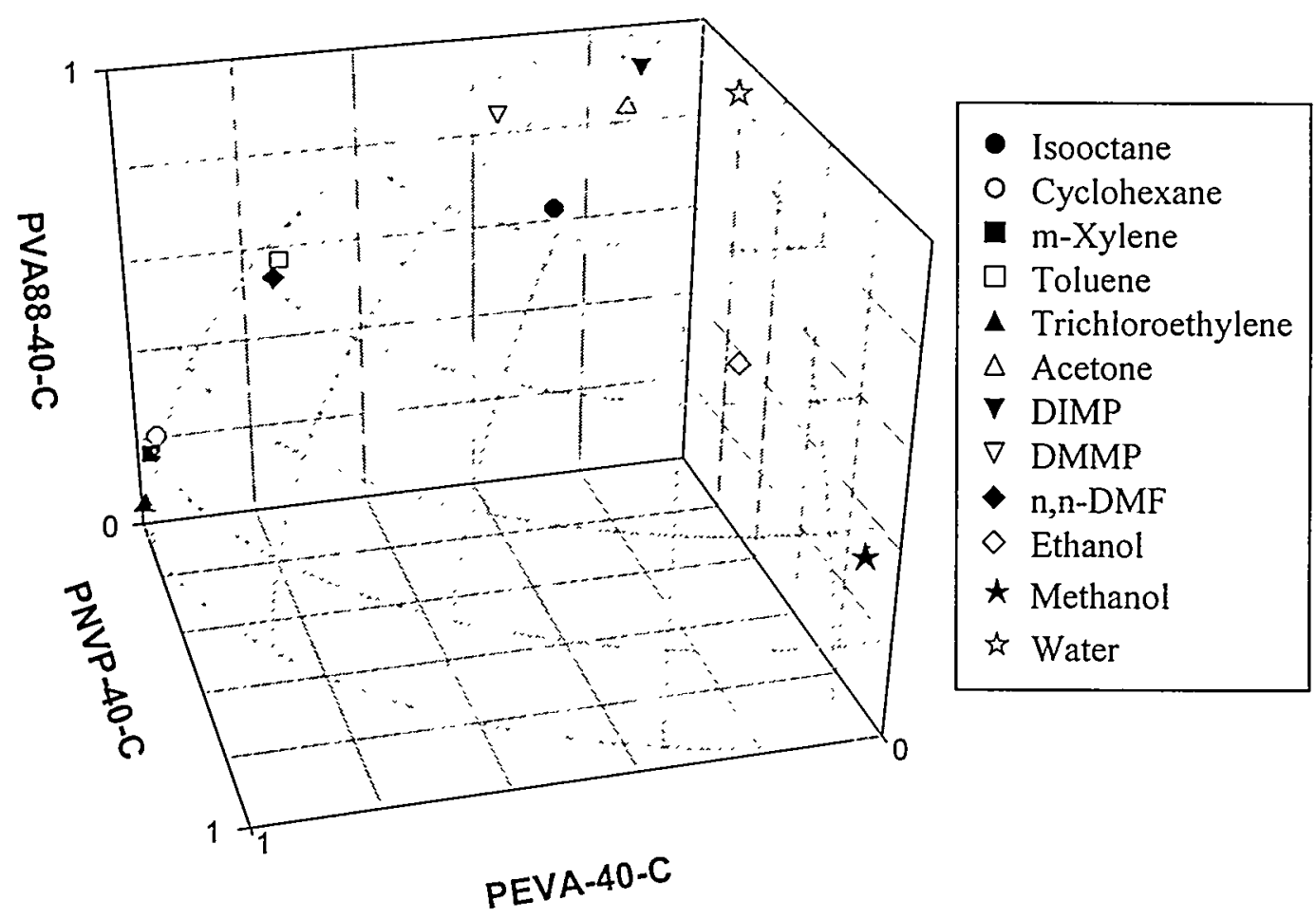

Figure 3 The same data as in Fig. 2 replotted for pattern recognition by transforming the response of each VOC to a 3-D vector equalized to fit on a unit sphere. Most of are easily identified in this fashion by their vector position on the sphere.

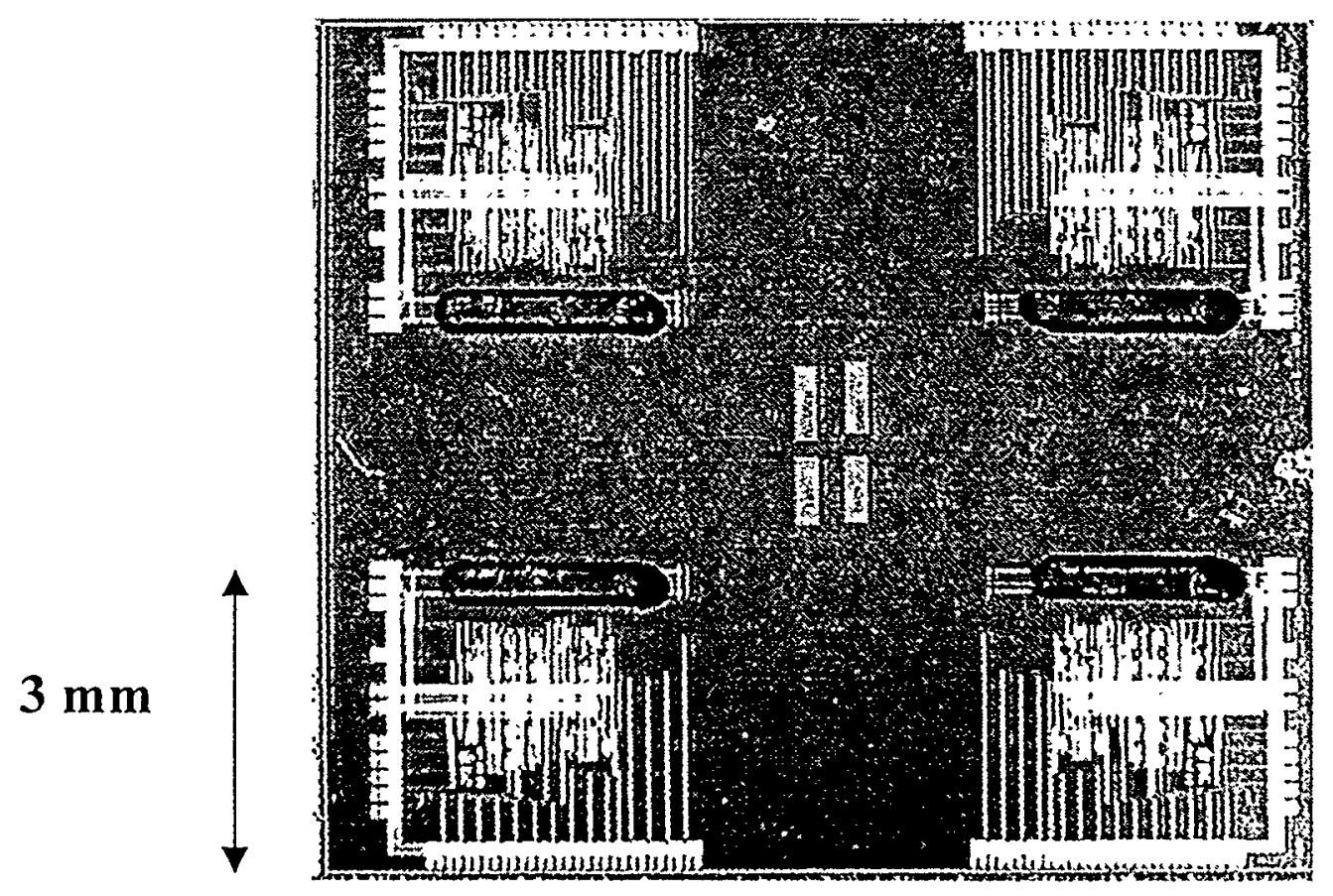

Figure 4 The Sandia Integrated Chemiresistor Array composed of four chemiresistors on their electrodes with the individual ASIC circuits for measuring the chemical responses and correcting for temperature changes. 


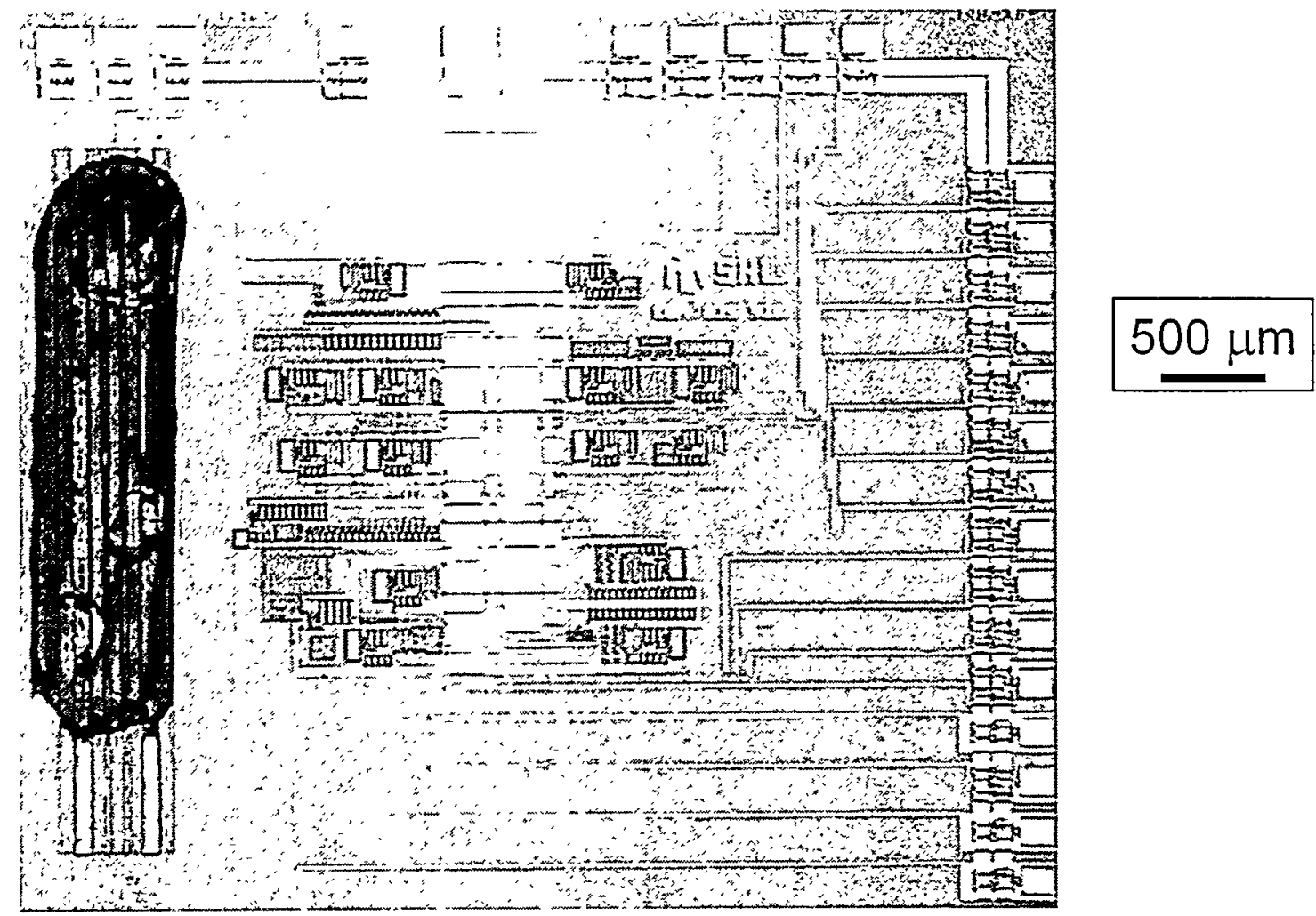

Figure 5 A close up of one of the ASICs showing the electrode pattern (Pt/Ti over Al by post-processing), the ASIC circuit elements and the bonding pads.

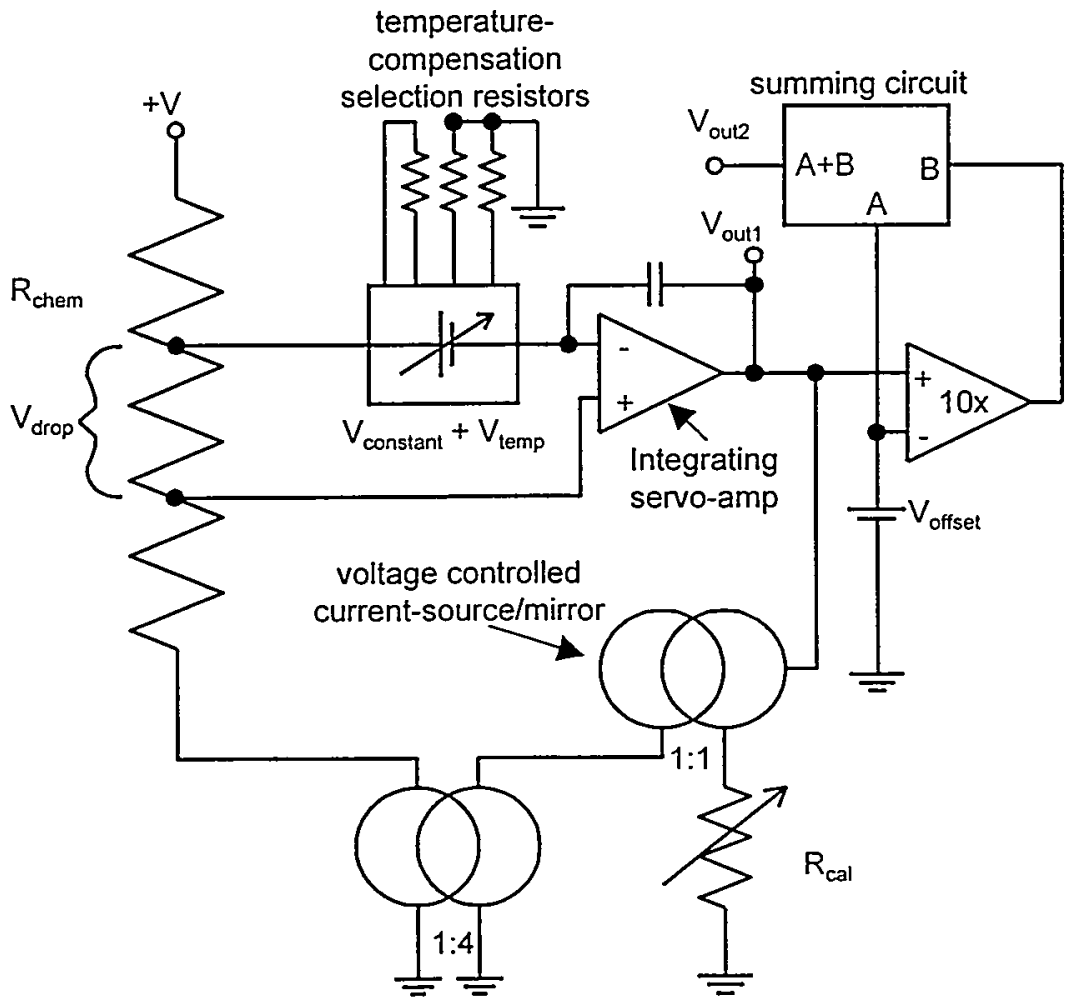

Figure 6 The circuit schematic for the ASIC. 


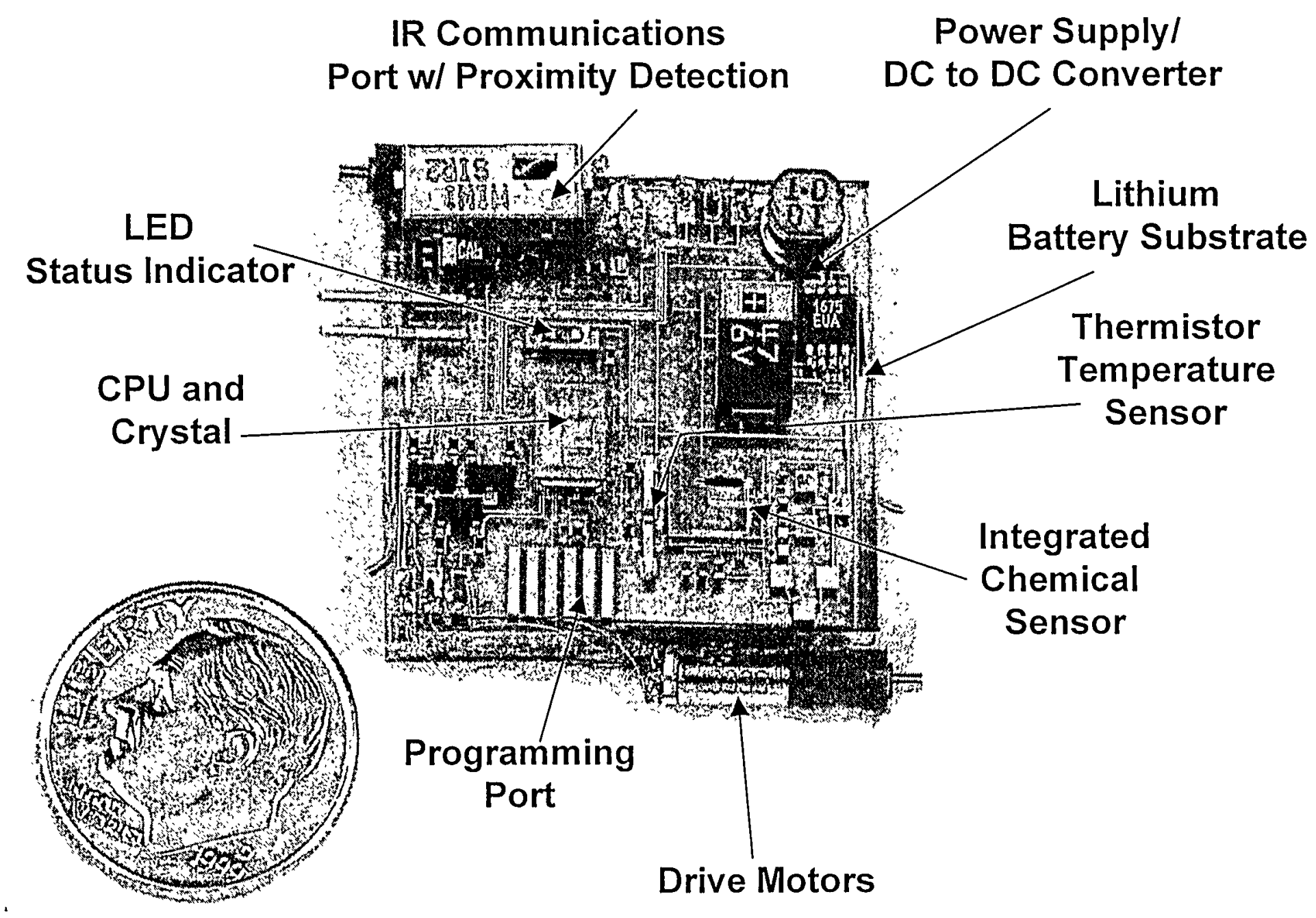

Figure 7 A prototype microrobot with the Integrated Chemiresistor Array wire bonded to the base PC board. The wheels have been removed from the drive motors for clarity. 